\title{
Effect of specific collagen peptides with various dosages on body composition in untrained men
}

\author{
D. Zdzieblik ${ }^{1}$, S. Oesser ${ }^{2}$, P. Dressler ${ }^{1}$, A. Gollhofer ${ }^{1}$ and D. König ${ }^{1}$ \\ ${ }^{1}$ Department of Sport and Sport Science, University of Freiburg, Freiburg, Germany and \\ ${ }^{2}$ CRI, Collagen Research Institute GmbH Kiel, Germany
}

As a result of the aging process muscle mass and function decreases. Accompanied by an increased fat mass this is highly associated with health complaints. Because of an aging society, interventions focusing on an improvement of body composition are an important effort in preventive medicine. Several clinical trials have shown that resistance training in combination with protein supplementation improves muscle mass and function by e.g. influencing the mTOR pathway ${ }^{(1)}$.

Recently, it has been demonstrated that specific collagen peptides (SCP) together with intense resistance exercise improved muscle strength and body composition in elderly sarcopenic men ${ }^{(2)}$. However, the dosage needed to induce respective effects is still not clear.

Therefore, the aim of this study was to investigate the effects of resistance exercise combined with different dosages of collagen peptides on body composition in middle-aged untrained men.

105 men aged 30 to 60 years performed resistance training three times per week for 12 weeks. Participants ingested 10,15 or $20 \mathrm{~g}$ of collagen peptides daily. Changes in fat free mass (FFM) and fat mass (FM) after supplementation of different dosages of specific collagen peptides (BODYBALANCE ${ }^{\circledR}$ ) was determined by DEXA, respectively. Differences within groups were statistical analyzed with Wilcoxon Rank-Sum test. Kruskal-Wallis-Test was used for differences between study groups.

\begin{tabular}{|c|c|c|c|c|c|}
\hline \multirow[b]{2}{*}{ Group } & \multirow{2}{*}{$\begin{array}{l}\text { Dosage } \\
\text { per day }\end{array}$} & \multicolumn{2}{|c|}{$\Delta \mathrm{FFM}(\mathrm{kg})$} & \multicolumn{2}{|c|}{$\Delta \mathrm{FM}(\mathrm{kg})$} \\
\hline & & Mean & SE & Mean & $\overline{\mathrm{SE}}$ \\
\hline $\mathrm{A}(\mathrm{n}=37)$ & $10 \mathrm{~g}$ & 1.96 & 1.59 & $-2 \cdot 04$ & $2 \cdot 71$ \\
\hline $\mathrm{B}(\mathrm{n}=33)$ & $15 \mathrm{~g}$ & $2 \cdot 34$ & $1 \cdot 36$ & $-2 \cdot 25$ & $2 \cdot 51$ \\
\hline$C(n=35)$ & $20 \mathrm{~g}$ & $2 \cdot 40$ & $1 \cdot 17$ & -2.75 & $2 \cdot 86$ \\
\hline
\end{tabular}

Values are means for the changes after 12 weeks of supplementation in 3

dosage groups. Mean values were not significantly different (Kruskal-Wallis): $* \mathrm{P}<0.05$

At the baseline level, no statistically significant differences between the study groups could be detected (data not shown). Collagen peptide supplementation led to an pronounced, statistically significant increase in s FFM ( $p<0.001)$ and a decrease in FM ( $<<$ 0.001). The results suggest a dose-dependent effect, although no statistical significant differences between groups regarding the FFM and FM changes (table) could be determined. Subjects receiving $10 \mathrm{~g}$ of SCP gained $19 \%$ less FFM than those with the intake of $15 \mathrm{~g}$ per day. In contrast a further increase of the daily SCP dosage ( $20 \mathrm{~g})$ resulted only in a $2.6 \%$ higher gain of FFM compared to the lower dosage of $15 \mathrm{~g}$ per day. This dose-depending effect of a SCP intake was also demonstrated for FM reduction. These results assume that resistance training combined with specific collagen peptide supplementation has a positive effect on body composition, whereby a daily post exercise intake of $15 \mathrm{~g}$ is recommended.

The study was conducted with the approval of the Ethics Committee of the Medical Faculty of the University of Freiburg. All participants gave written informed consent.

1. Cermak NM, Res PT, de Groot LCPGM et al. (2012) Am J Clin Nutr 96, 1454-64.

2. Zdzieblik D, Oesser S, Baumstark MW et al. (2015) Br J Nutr 114, 1237-45. 